\title{
Estudio descriptivo de la educación religiosa de estudiantes de secundaria de la provincia de Córdoba (España)
}

Sonia García-Segura

Universidad de Córdoba (España)

María José Martínez Carmona

Universidad de Córdoba (España)

Carmen Gil del Pino

Universidad de Córdoba (España) 



\title{
Estudio descriptivo de la educación religiosa de estudiantes de secundaria de la provincia de Córdoba (España)
}

\section{Descriptive study of religious education of secondary students of the province of Cordoba (Spain)}

\author{
Sonia García-Segura \\ Universidad de Córdoba (España) \\ sgsegura@uco.es
}

María José Martínez Carmona

Universidad de Córdoba (España)

mjmartinez@uco.es

Carmen Gil del Pino

Universidad de Córdoba (España)

edlgipim@uco.es

Fecha de recepción: 10 de octubre de 2018

Fecha de aceptación: 5 de abril de 2020

\section{Resumen}

El fenómeno de la globalización ha trasformado nuestros centros educativos en entornos plurales. La diversidad cultural se concentra y convive en ellos, y las creencias religiosas, que se aprenden dentro de las culturas, constituyen una cuestión social y política, de primer orden, por lo que deben ser atendidas de manera prioritaria en la investigación científico-social, así como sus implicaciones educativas. El presente trabajo de investigación tiene como objetivo descifrar primero y describir después el pensamiento implícito de los jóvenes sobre la diversidad religiosa y la enseñanza de la religión en España utilizando como base el proyecto europeo REDCo (Religion in Education. A contribution to dialogue or a factor of conflict in transforming societies of European Countries), proyecto financiado por el Departamento de Investigación de la Comisión Europea para establecer las potencialidades y limitaciones de la religión en los sistemas educativos de ocho países seleccionados, a saber, Estonia, Rusia, Noruega, Alemania, Países bajos, Inglaterra, Francia y España. El instrumento utilizado para la recogida de información es un cuestionario validado por REDCo. Se implementó a 385 estudiantes de cuatro centros de Educación Secundaria de Córdoba (España) caracterizados por su compleja y líquida diversidad de creencias. Los análisis descriptivos señalan, primero, el interés del alumnado por conocer otras religiones 
y/o concepciones del mundo a través de agrupaciones flexibles; segundo, la existencia de diferentes experiencias relacionadas con la enseñanza de la religión y, tercero, su posición dispareja respecto a los modelos didácticos empleados en la enseñanza de la religión.

Palabras clave: Religión; Diversidad cultural; Educación religiosa; Minorías religiosas; Córdoba (España)

\begin{abstract}
The phenomenon of globalisation has transformed our educational centres into plural environments. Cultural diversity is concentrated and coexists in them; and religious beliefs, which are learned within cultures, constitute a major social and political issue which must be therefore addressed as a priority in scientific-social research, as well as its educational implications. This aim of this research work is to figure out and then describe the implicit thinking of young people about religious diversity and the teaching of religion in Spain, using the European project REDCo (Religion in Education: A contribution to dialogue or a factor of conflict in transforming societies of European Countries) as a basis. This project is funded by the Research Department of the European Commission and is intended to establish the potentialities and limitations of religion in the educational systems of eight different countries, namely Estonia, Russia, Norway, Germany, Netherlands, England, France and Spain. The instrument used for the collection of data has been a questionnaire validated by RDCo. It was administered to 385 students from four Secondary Education centres in Córdoba (Spain), characterised by their complex and changing diversity of beliefs. The descriptive analyses indicate, first, the interest of students to understand other religions and/or worldviews through flexible grouping; second; the existence of different experiences with religion in the school and, third; the varying positions of students with respect to the different models of teaching religion.
\end{abstract}

Keywords: Religion; Cultural diversity; Religious education; Religious minorities, Cordoba (Spain)

\title{
1. INTRODUCGIÓN
}

La enseñanza confesional es un hecho consolidado en las escuelas públicas de gran parte de los países de Europa. Mientras que en unos como Reino Unido, Suecia, Finlandia, Alemania, Austria, Grecia, Luxemburgo, Suiza o Rumanía es obligatoria (con posibilidad de exención), en otros solo lo es para los centros, que han de garantizar su oferta, siendo opcional para los alumnos. Es el caso de España, Holanda, Bélgica, Italia o República Checa.

La formación religiosa está, pues, garantizada en la mayor parte de los Estados de la Unión Europea, ya sean confesionales o no confesionales. Estos últimos han suscrito convenios con las distintas iglesias arraigadas en sus territorios en los que se estipula que estas impartan su doctrina en las escuelas públicas responsabilizándose de los aspectos curriculares y que los Estados sufraguen los gastos derivados de dicha enseñanza. 
Por lo que respecta a España, La Constitución vigente dispone, en su artículo 16.3, que ninguna confesión tendrá carácter estatal, pero los poderes públicos considerarán las creencias religiosas y mantendrán relaciones de cooperación con las distintas confesiones. Y en el artículo 27.3 establece que se garantizará "el derecho que asiste a los padres para que sus hijos reciban la formación religiosa y moral que esté de acuerdo con sus propias convicciones".

El acuerdo entre el Estado Español y la Santa Sede fue aprobado en 1979. El artículo II de mismo determina que "los planes educativos [...] incluirán la enseñanza de la religión católica en todos los centros de educación, en condiciones equiparables a las demás disciplinas fundamentales". Claro que el pacto también regula el derecho de los estudiantes a no recibirla:

Por respeto a la libertad de conciencia, dicha enseñanza no tendrá carácter obligatorio para los alumnos. Se garantiza, sin embargo, el derecho a recibirla. Las autoridades académicas adoptarán las medidas oportunas para que el hecho de recibir o no recibir la enseñanza religiosa no suponga discriminación alguna en la actividad escolar (art. 2).

Y una de las medidas adoptadas fue la de las clases separadas, aunque la creciente realidad multicultural y multirreligiosa aparecida en España ha conducido forzosamente, como afirma López González (2012, p.64), "al ensayo de enfoques formativos de tipo intercultural e interreligioso", cuyos resultados favorables han estimulado experiencias que contemplan el diálogo bien de forma transversal bien integrada en la materia Valores éticos.

\section{FUNDAMENTOS TEÓRICOS}

\subsection{Diversidad cultural y religiosa}

En un mundo cada vez más globalizado, lo uniforme y homogéneo ha dado paso a lo plural y complejo. El fenómeno de la inmigración ha roto la imagen de una sociedad consistente apuntalada en torno a un único sistema de valores y patrones de conducta. La actual es una sociedad pluralista en la que distintas culturas presentan modelos de vida y de pensamiento diferentes (Banks, 2009; Pedone, 2013). Se trata de la sociedad del mosaico, compuesta por numerosos grupos culturales que producen una socialización primaria multicultural, multirreligiosa y multilingüe. Diferentes culturas se citan hoy en un mismo espacio social y escolar buscando expresarse, respetarse, complacerse y entrelazarse (Banks, 2009).

Asistimos, pues, al surgimiento de un mundo diverso con evidente reflejo en el terreno de la educación. Ciertamente se han puesto en marcha prácticas pedagógicas que contemplan las múltiples manifestaciones latentes durante tantos años y los sentimientos vivos de identidad cultural y religiosa. Y es que no solo ha cambiado 
el rol del profesor sino también el marco normativo de la escuela (Mijares, 2006). Esta debe promover ahora nuevos modelos interculturales e interreligiosos que proporcionen una educación de calidad para todos. Los distintos gobiernos han dispuesto medidas y recursos para atender a la diversidad del alumnado y favorecer su inclusión educativa y social (Fernández Echeverría y García Castaño, 2015).

En el caso de España, destino principal de inmigrantes provenientes del norte de África, América del Sur y este de Europa, se han ido generando múltiples expresiones de tipo cultural, lingüístico, ideológico, etcétera. Ciertamente, la sociedad española es cada vez más plural a todos los niveles. Por lo que atañe a la religión, que es lo que nos compete en este trabajo, la diversidad es clara, así como la conciencia de ella.

Por lo que atañe a las escuelas, la presencia de alumnado no autóctono ha dejado de ser un hecho coyuntural para convertirse en uno de carácter estructural. Se ha producido un creciente flujo de alumnos entre finales de la década de los noventa y el año 2010, momento en que se detuvo a causa de la crisis económica. En 2012, el número de estudiantes procedentes de otros países en las escuelas españolas se situaba, según el Ministerio de Educación, Cultura y Deporte (2013), en un 9.43\% del total. El final de la crisis ha supuesto un nuevo cambio en los movimientos migratorios de nuestro país. Ello queda reflejado en los datos estadísticos del curso escolar 2016/2017 (Ministerio de Educación, Cultural y Deporte, 2017), en los que se comprueba que el porcentaje de alumnado extranjero ha disminuido casi un punto, situándose en un $8.5 \%$ sobre el número total.

En cuanto a la distribución de los estudiantes en función de la alternativa de formación religiosa que eligen (Católica, Evangélica, Islámica, Judía e Historia y Cultura de las Religiones), durante el curso escolar 2015-2016, según el Ministerio de Educación y Formación profesional (2018), un 66.7\% de alumnado de Educación Primaria escolarizado en España recibía educación religiosa, porcentaje que desciende a $54.35 \%$ si nos situamos en la Educación Secundaria Obligatoria y a $29.82 \%$ si lo hacemos en Bachillerato, siendo la religión católica la más elegida en todos los casos. El porcentaje de "otras religiones" tan solo era de $0.84 \%$ en Educación Primaria, 1.26\% en Secundaria Obligatoria y $0.02 \%$ en Bachillerato.

En el caso de Andalucía, durante ese mismo curso 2015-2016, tan solo cursaba "otras religiones" un 1.2\% del alumnado de Educación Primaria, un 0.6\% de Secundaria Obligatoria y un $0.0 \%$ de Bachillerato.

\subsection{Del conflicto interreligioso al diálogo}

En el ser humano existe una tendencia poderosa a rechazar lo ajeno, lo extraño, "lo otro", y a convertirlo en problema (Ortega Ruiz, 2004). Los tópicos con los que nos referimos a los "distintos" confirman ideas, costumbres y actitudes larvadas de repulsa hacia ellos construidas socialmente, políticamente, que operan en nosotros fijándonos los límites de lo que es aceptable o inaceptable. 
Los estereotipos son convicciones profundas a través de las que interpretamos a los otros. Instaladas en el estrato más básico de nuestra arquitectura mental, se entremezclan con temores, intereses, proyecciones, actitudes..., y se traducen en conductas de rechazo y discriminación desde una supuesta defensa de las propias formas de vida y de cultura.

En materia de religión, la expresión de los múltiples credos se aceptará o proscribirá en función de procesos políticos y sociales reguladores no solo explícitos sino también implícitos. En el trabajo de Briones (2010) se pone de manifiesto el pluralismo religioso en nuestro contexto más cercano y la importancia del diálogo para establecer una necesaria "interreligiosidad" en estas sociedades cada día más globalizadas.

En su preámbulo, la Constitución de la UNESCO (2014) proclama lo siguiente: "puesto que las guerras nacen en la mente de los hombres, es en la mente de los hombres donde deben erigirse los baluartes de la paz" (p.7). Esta se edifica, pues, en el pensamiento, pero también en la voluntad, en el sentimiento y en la conducta de los seres humanos, dado que son todos integrales.

En el panorama actual, tan diverso como convulso, la paz es un imperativo categórico, lo que requiere expandir los marcos de referencia propios y entender los de los demás o dicho de otro modo, abrirse al conocimiento y reconocimiento de las creencias ajenas. Tal apertura constituye la base de una cultura de diálogo que haga posible la cohesión social en un mundo globalizado (Castilla, 2011).

Para Álvarez Castillo, González González y Fernández Caminero (2012), los principios básicos del diálogo interreligioso son la capacidad de escucha, el respeto a la pluralidad de creencias, la identificación de la experiencia religiosa común y la apertura a la experiencia diferente. Todos ellos constituyen el marco idóneo para que la pluralidad religiosa deje de ser un problema y se convierta en un valioso recurso educativo para el logro de una coexistencia pacífica basada en el respeto de la dignidad humana independientemente de las convicciones religiosas.

\subsection{El proyecto REDCo}

El panorama descrito anteriormente ha propiciado un encendido debate en la sociedad española que refleja la tensión existente entre dos posturas: el reconocimiento de la libertad de creencias de los miembros de la comunidad educativa y el respeto a una escuela laica contextualizada en un escenario de pluralismo doctrinal. Ello pone de relieve la necesidad de situar el tema religioso en un lugar destacado de la agenda de los estudiosos de la educación, más aún cuando evidenciamos que, en nuestro país, son escasas las investigaciones realizadas al respecto, sobre todo las centradas en la etapa de Educación Secundaria. Sin embargo, en el contexto europeo sí hemos hallado estudios sobre el tema en los que se ha conseguido información de los estudiantes 
fundamentada en sus percepciones, creencias, opiniones, significados y actitudes respecto a la religión dentro y fuera del ámbito educativo (Kay \& Ziebertz, 2006).

En este marco nació el proyecto Religion in Education: A contribution to dialogue or a factor of conflict in transforming societies of European Countries, (REDCo), en el que han colaborado las siguientes universidades europeas: Hamburgo (Alemania), Stavanger (Noruega), Warwick (Reino Unido), Tartu (Estonia), San Petersburgo (Rusia), Utrecht (Países Bajos), Paris-Sorbonne (Francia) y Granada (España). Desarrollado entre 2006 y 2009, perseguía establecer el rol, las posibilidades y las limitaciones de la religión en los sistemas educativos de los ocho países y descubrir la opinión que sobre su enseñanza tenían los jóvenes de entre 14 y 16 años (Weisse, 2009) así como la forma en que estos percibían la diversidad religiosa tanto dentro como fuera del ámbito educativo (Valk; Bertram $\square$ Troost; Friederici \& Beredrd, 2009). No interesaban los sistemas de creencias en abstracto sino las formas reales de religión y las cosmovisiones explicitadas por los propios jóvenes objeto de estudio.

Una vez concluido el proyecto REDCo, y dados los resultados positivos que se obtuvieron (European Commission, 2009), se puso en marcha, en 2012, una segunda fase del mismo denominada REDCo II, en la que se planteó realizar un estudio cuantitativo en once países europeos (Inglaterra, Estonia, Francia, Alemania, Países Bajos, Noruega, Rusia, España, Suecia, Finlandia y Ucrania) y en Suráfrica y México (Dietz; Rosón y Ruiz, 2011; Jackson, 2012; Bertram-Troost; Schihalejev \& Neill, 2015). Es aquí donde halla cobertura el presente trabajo.

\subsection{Contexto de la investigación: Córdoba, crisol de culturas y religiones}

Córdoba es mundialmente reconocida como un modelo de paz y convivencia, un paradigma de civilización humana y de unidad moral, y ello debido a la sabiduría que rezuma por sus cuatro costados y a su trayectoria histórica de inmensa pluralidad. No en vano es cuna de Séneca, Averroes y Maimónides y concilio ejemplar de credos.

Es sabido por todos que la Córdoba medieval era un espacio impregnado por la cultura greco-romana en el que convivían plácidamente cristianos, judíos y musulmanes. En ella se congregaba un riquísimo acervo de valores con distintos orígenes culturales o religiosos. La libertad de creencias, el respeto y el reconocimiento mutuos, el diálogo creativo, la armonía entre religiones, la idea de una humanidad común, la verdad, la solidaridad, etcétera, constituían la atmósfera exuberante de aquella ciudad única en el universo.

Ahora bien, el pasado de Córdoba no es un asunto concluido. Este ofrece al ciudadano de hoy directrices útiles para avanzar en el proceso de alojamiento y aceptación mutuos y para construir una sociedad inclusiva basada en un debate intercultural e interreligioso entre identidades flexibles abierto y con puntos de vista integradores. Aquí reside la razón de elegir este contexto para nuestro estudio. La 
herencia cultural de una Córdoba remota inigualable que fue símbolo de diversidad ha germinado y florecido en un presente que nos invita con insistencia a indagar sobre el estado de las cosas.

\section{OBJETIVOS DEL ESTUDIO}

El objetivo primero y primordial del estudio es, por un lado, describir el papel que para los jóvenes cordobeses tiene la religión dentro de la escuela y, por otro, descubrir sus percepciones sobre la diversidad religiosa en las aulas, objetivo que se concreta en los específicos que exponemos a continuación:

- Conocer con exhaustividad el perfil del alumnado que cursa Educación Secundaria Obligatoria y su relación con la religión.

- Recabar información sobre la percepción del alumnado de Educación Secundaria Obligatoria en cuanto a la religión en la escuela, a saber, sus experiencias, su opinión respecto a distintos posicionamientos de la escuela ante la religión, la ayuda que a su juicio proporciona su aprendizaje y su posición respecto a los distintos modelos de enseñanza en la escuela.

- Descubrir el tipo de agrupamiento que el alumnado considera de mayor interés para la enseñanza de la religión como modalidad de atención a la diversidad.

\section{METODOLOGÍA}

\subsection{Diseño de la investigación}

El presente estudio descriptivo utiliza un cuestionario como instrumento de medida en la investigación partiendo de una perspectiva cuantitativa de carácter flexible y adaptada al contexto de aplicación. Se distinguen datos de carácter clasificatorio de la muestra y datos referidos a las opiniones sobre la realidad de la religión en la escuela, para lo cual se alternan preguntas estructuradas con respuesta cerrada con otras de respuesta libre y escalas de tipo Likert con 5 grados de elección, según la necesidad de respuesta.

Tras la recogida de los datos, se codifican las respuestas recibidas y se vuelcan en una matriz de datos que permite el análisis a través del programa estadístico SPSS v. 21.0. Una vez hallada la fiabilidad del cuestionario por medio del análisis de constitución interna Alpha de Cronbach, se evalúa el funcionamiento de las variables de modo descriptivo a través de medias aritméticas y desviación típica, para conocer así los datos de contexto y de entrada o input. Finalmente, se procede a contrastar los valores predictivos a través de un ANOVA de medidas repetidas en cuatro variables intrasujetos, según su distribución normal. 


\subsection{Instrumento}

El Cuestionario sobre Religión y Educación utilizado ha sido previamente validado por REDCo e implementado en otras regiones europeas y españolas como Granada, Melilla y Murcia (Dietz, Rosón y Garzón, 2011). Dicho cuestionario está compuesto por diferentes ítems distribuidos en cuatro bloques, uno preliminar de carácter sociodemográfico destinado a extraer datos para la descripción de la muestra y tres de contenido específico del ámbito de estudio: el primero de ellos se denomina Religión en la escuela; el segundo bloque conforma Tú y la religión; y el último tiene por título Tú y los demás.

\subsection{Descripción de la muestra}

La muestra del presente estudio está integrada por 385 sujetos que presentan las siguientes características:

- Sus edades están comprendidas entre los 12 hasta los 18 años, si bien el $74.2 \%$ ( $\mathrm{n}=$ 286) tiene entre 14 y 15.

- El 49.1\% son hombres $(n=189)$ y el $50.9 \%$ mujeres $(n=196)$.

- Provienen de cuatro centros de Educación Secundaria de Córdoba y provincia, tres públicos y uno concertado: el IES Aljanadic de Posadas ( $\mathrm{n}=105,27.3 \%)$, el IES Alhakén II de Córdoba capital $(\mathrm{n}=131,34 \%)$, el IES Mario López de Bujalance ( $\mathrm{n}$ $=93,24.2 \%)$ y el Colegio Santísima Trinidad de Córdoba $(\mathrm{n}=56,14.5 \%)$. Se trata de centros grandes, con varias líneas en Educación Secundaria, estudios de Grado Medio, Grado Superior y Bachillerato.

- Todos se encuentran cursando Educación Secundaria Obligatoria: algo más de la mitad tercercurso $(n=199,51.7 \%)$ y el resto cuarto $(n=112,29.1 \%)$ y segundo $(n=74,19.2 \%)$.

- El país de nacimiento de la mayoría de ellos es España $(97.4 \%, \mathrm{n}=375)$, aunque también hay quien procede de países como Alemania, Argelia, Bolivia, Colombia, Ecuador, México, Nicaragua y Venezuela, con porcentajes que en ningún caso superan el $0.5 \%$. De igual modo, el país de nacimiento de la madre es por lo general España $(95.4 \%, \mathrm{n}=367)$, si bien se registran casos de nacidas en Venezuela, Nicaragua, Italia, Francia, Ecuador, Camboya, Bolivia, Argelia, Alemania y Colombia (con valores inferiores a $0.5 \%$, salvo en el último caso, que asciende al $1.3 \%, \mathrm{n}=5$ ). La respuesta es semejante en el caso de los padres. El 95.8\% nació en España ( $\mathrm{n}=$ 369) y el resto en Alemania, Argelia, Australia, Bolivia, Colombia, Ecuador, Francia, Marruecos, México, Nicaragua, Senegal y Argelia, con valores máximos de $0.8 \%$ (en el caso de Alemania).

- La nacionalidad del $97.7 \%$ de la muestra $(\mathrm{n}=376)$ es la española. La del resto es nicaragüense, venezolana, ecuatoriana $\mathrm{o}$, en un porcentaje muy pequeño $(0,3 \%)$, doble: española y colombiana, española y alemana, española y argelina, española y colombiana, española y ecuatoriana y, finalmente, española y francesa. 
- El idioma más usado en casa es el español, que, en frecuencias muy bajas, en ningún caso por encima del $0.3 \%$, se combina con el inglés, el italiano, el alemán, el árabe, el camboyano, el catalán o el francés.

- El 43.9\% $(n=169)$ de la muestra indica que su progenitor tiene una religión o concepción del mundo definida, el $28.6 \%(n=110)$ expresa que no y el $27.5 \%$ $(\mathrm{n}=106)$ que no lo sabe. En los casos afirmativos, el alumnado indica que los padres son ateos $(2.4 \%, \mathrm{n}=9)$, de religión católica $(39.7 \%, \mathrm{n}=151)$, católica y evangélica y evangélica o musulmana (en los últimos tres casos con porcentajes inferiores a 1.8). Asimismo, la respuesta ante la misma pregunta en cuanto a la madre es afirmativa en el $52.7 \%$ de los casos $(n=203)$, negativa en el $21 \%(n=81)$ y desconocida en el $26.2 \%(n=101)$. En el primer caso, los alumnos han indicado respecto a la concepción del mundo que su madre es atea $(1.5 \%, \mathrm{n}=6)$, católica $(48.1 \%, \mathrm{n}=185)$, evangélica $(2.1 \%, \mathrm{n}=8) \mathrm{y}$, en menor medida, budista, hindú, católica y evangélica.

- Por lo que respecta a la concepción del mundo o religión de los propios sujetos de la muestra, el $77.9 \%,(n=300)$ declara tener una religión o concepción del mundo, siendo la católica romana en un $59.9 \%$ de los casos $(\mathrm{n}=219)$, ateísmo $(11.2 \%, \mathrm{n}=$ $43)$, evangélica $(4.4 \%, \mathrm{n}=17) \mathrm{y}$, en menor medida, islámica, hindú o budista. El $4.2 \%$ del alumnado indica otra religión o confesión $(n=16)$.

- En cuanto a los años que el alumnado ha estudiado religión en la escuela (tabla 1), solo el 13.5\% manifiesta haberlo hecho menos de 7 años, siendo este el número de años con mayor porcentaje de respuesta $(\mathrm{n}=91 ; 23.6 \%)$. No obstante, el porcentaje del alumnado que afirma estar asistiendo a clases de religión $(n=223,57.9 \%)$ es levemente superior al de quienes no asisten $(n=162,42.1 \%)$.

Tabla 1. Años que el alumnado ha estudiado religión en la escuela

\begin{tabular}{cccccccccccccccc}
\hline \multicolumn{11}{c}{ ¿Cuántos años has estudiado religión en la escuela? } \\
\cline { 2 - 15 } & 1 & 2 & 3 & 4 & 5 & 6 & 7 & 8 & 9 & 10 & 11 & 12 & 13 & 14 & 16 \\
\hline $\mathrm{n}$ & 22 & 3 & 4 & 9 & 8 & 6 & 91 & 21 & 46 & 59 & 65 & 12 & 16 & 21 & 2 \\
\hline$\%$ & 5.7 & .8 & 1 & 2.3 & 2.1 & 1.6 & 23.6 & 5.5 & 11.9 & 15.3 & 16.9 & 3.1 & 4.2 & 5.5 & .5 \\
\hline
\end{tabular}

\section{ANALISIS DE LOS RESULTADOS}

Una vez analizados los datos de contexto, en esta primera parte del cuestionario, el planteamiento de partida es conocer los datos de entrada o input. Con este fin, los ítems son agrupados en torno a los siguientes componentes principales: las experiencias del alumnado con la religión en la escuela, su opinión respecto a distintos posicionamientos de la escuela ante la religión, la ayuda que, desde su punto de vista, proporciona su aprendizaje y, por último, su posición respecto a los distintos modelos de enseñanza en la escuela. En todos ellos se han planteado afirmaciones 
con las que el alumnado, a través de una escala tipo Likert, ha mostrado su grado de acuerdo, siendo 1 "totalmente de acuerdo" y 5 "estoy en total desacuerdo". Tras el cálculo de la consistencia interna, los coeficientes de fiabilidad en los distintos componentes (alpha de Cronbach) fueron .693, .678, .853, .723, siguiendo el orden. Estos indican que la consistencia interna del constructo es adecuada, por lo que se mantiene su uso para los análisis críticos.

\subsection{Religión en la escuela}

A continuación, presentamos los resultados registrados tras un análisis descriptivo de las experiencias que el alumnado ha tenido en la escuela. La siguiente tabla (2) muestra que este se halla muy de acuerdo con la idea de que en clase aprende a respetar a todos, independientemente de su religión, con una media de respuesta de $1.66(\mathrm{~N}=385)$. En este sentido, el $61,7 \%$ del alumnado ha mostrado algún grado de acuerdo. Asimismo, se muestra bastante de acuerdo con las afirmaciones de que en la escuela obtiene conocimiento sobre las diferentes religiones (el 56.1\% se muestra totalmente de acuerdo) y tiene oportunidad de hablar sobre cuestiones de religión desde diferentes perspectivas $(M=2.42)$. En cambio, su posición es neutral respecto a que los temas que se tratan en religión son interesantes o a que aprender sobre la religión en general le ayude a aprender sobre sí mismo $(\mathrm{M}=2.96$ y 2.85 , respectivamente). Por otra parte, el $66 \%$ no se muestra de acuerdo o está en total desacuerdo con la afirmación de que aprender sobre religiones genera conflictos en el aula $(n=254)$.

Tabla 2. Respuesta registrada del alumnado de acuerdo con sus experiencias con la religión en la escuela

\begin{tabular}{lcrc}
\hline & M & DT & N \\
\hline En la escuela obtengo conocimiento sobre las diferentes religiones. & 2,31 & 1,11 & 384 \\
\hline En la escuela aprendo a respetar a todos, independientemente de su religión. & 1,66 &, 93 & 385 \\
\hline $\begin{array}{l}\text { En la escuela tengo oportunidad de hablar sobre cuestiones de religión desde } \\
\text { diferentes perspectivas. }\end{array}$ & 2,42 & 1,15 & 385 \\
\hline Los temas que tratan sobre religión en la escuela son interesantes. & 2,96 & 1,08 & 385 \\
\hline Aprender sobre la religión en general me ayuda a aprender sobre mí mismo. & 2,85 & 1,19 & 385 \\
\hline Aprender sobre religiones genera conflictos en el aula. & 3,89 & 1,12 & 385 \\
\hline
\end{tabular}

5.2. Opinión respecto a distintos posicionamientos de la escuela ante la religión

Por lo que atañe al posicionamiento del alumnado respecto a las manifestaciones de la religión en la escuela y el ejercicio de la autoridad en esta (ver tabla 3), obtenemos puntuaciones centrales en cuanto al hecho de llevar a la escuela símbolos 
religiosos visibles (por ejemplo, velos), con una media de 3.17. Si embargo, los valores arrojados muestran que no hay consenso de respuesta en relación con poder llevar símbolos religiosos discretos (por ejemplo, cruces pequeñas colgadas en el cuello). Un 24.9\% ( $\mathrm{n}=96)$ no está de acuerdo ni en desacuerdo, si bien el 31.7\% ( $\mathrm{n}=$ $122)$ está totalmente de acuerdo frente al $14 \%(\mathrm{n}=54)$ que está en total desacuerdo. Los resultados revelan la necesidad de indagar más en este sentido.

Tabla 3. Respuesta registrada del alumnado de acuerdo con las diferentes manifestaciones de la religión en la escuela

\begin{tabular}{|c|c|c|c|}
\hline & $\mathbf{M}$ & D.T. & $\mathbf{N}$ \\
\hline $\begin{array}{l}\text { En el comedor de la escuela deben ser tomadas en cuenta las normas } \\
\text { alimenticias de cada religión. }\end{array}$ & 2,51 & 1,40 & 385 \\
\hline $\begin{array}{l}\text { Los alumnos/as deberían poder llevar símbolos religiosos en la escuela... } \\
\text { discretos (ej. cruces pequeñas, etc. colgadas en el cuello). }\end{array}$ & 2,89 & 4,53 & 385 \\
\hline $\begin{array}{l}\text { Los alumnos/as deberían poder llevar símbolos religiosos en la escuela más } \\
\text { visibles (ej. velos, etc. ). }\end{array}$ & 3,17 & 1,28 & 385 \\
\hline Los alumnos/as pueden ausentarse de la escuela durante sus fiestas religiosas. & 2,87 & 1,31 & 385 \\
\hline $\begin{array}{l}\text { Los alumnos/as deberían ser excusados de asistir a ciertas clases por razones } \\
\text { religiosas. }\end{array}$ & 3,51 & 1,21 & 385 \\
\hline $\begin{array}{l}\text { La escuela debería proporcionar espacios para que los alumnos puedan rezar en } \\
\text { la escuela. }\end{array}$ & 3,30 & 1,19 & 385 \\
\hline $\begin{array}{l}\text { Actos religiosos voluntarios (ej. oraciones) podrían formar parte de la vida de la } \\
\text { escuela. }\end{array}$ & 3,18 & 1,20 & 385 \\
\hline
\end{tabular}

La postura de los estudiantes tiende a la neutralidad también en referencia a que los actos religiosos voluntarios (por ejemplo, las oraciones) puedan formar parte de la vida académica $(\mathrm{M}=3.18)$ y a que la escuela proporcione espacios para que puedan rezar en ella $(\mathrm{M}=3.3)$. Sin embargo, el $37.9 \%$ no está de acuerdo o está en total desacuerdo $(\mathrm{n}=146)$. Menos identificado se siente el alumnado con la afirmación de que debería ser excusado de asistir a ciertas clases por razones religiosas $(\mathrm{M}=3.51)$, con un porcentaje de 52.2 que está en desacuerdo o en total desacuerdo $(\mathrm{n}=202)$. No obstante, hay consenso en que en el comedor escolar deben ser tomadas en cuenta las normas alimenticias de cada religión $(\mathrm{M}=2.51)$, con un $31.2 \%$ de los estudiantes totalmente de acuerdo $(\mathrm{n}=102)$.

5.3. Ayuda que, desde su punto de vista, proporciona la religión en su aprendizaje

Por otra parte, y como puede verse en la siguiente tabla (4), el alumnado se muestra de acuerdo con el hecho de que aprender sobre religiones ayuda a entender a los demás con el fin de convivir pacíficamente con ellos (con un porcentaje acumulado de $73 \%$ de acuerdo, $n=281)$, aprender sobre su propia religión $(M=2.08)$, 
desarrollar su propio punto de vista $(\mathrm{M}=2.12)$, comprender la historia (el $69.6 \%$ muestra algún grado de acuerdo, $n=268)$, desarrollar valores morales $(\mathrm{M}=2.24) \mathrm{y}$ obtener mejor comprensión de la actualidad (el 58.4\% muestra acuerdo, $\mathrm{n}=224$ ).

Tabla 4. Respuesta registrada del alumnado de acuerdo con la ayuda adquirida en su aprendizaje sobre religiones

\begin{tabular}{lccc}
\hline & M & D.T. & N \\
\hline Entender a los demás con el fin de convivir con ellos pacíficamente. & 2,02 & 1,01 & 385 \\
\hline Comprender la historia. & 2,23 & 1,08 & 385 \\
\hline Obtener mejor comprensión de la actualidad. & 2,38 & 1,09 & 385 \\
\hline Desarrollar mi propio punto de vista. & 2,12 & 1,04 & 385 \\
\hline Desarrollar valores morales. & 2,24 & 1,05 & 385 \\
\hline Aprender sobre mi propia religión. & 2,08 & 1,10 & 385 \\
\hline
\end{tabular}

5.4. Posición respecto a los distintos modelos de enseñanza en la escuela

Respecto a cómo se identifica el alumnado con los diferentes modelos de enseñanza de religión en la escuela (ver figura 1), está de acuerdo con la afirmación de que esta debería ser materia opcional (el 62.1\% está totalmente de acuerdo, n=239).

Estoy en total desacuerdo

No estoy de acuerdo

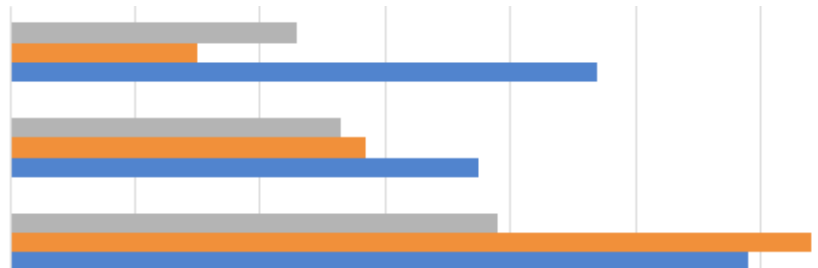

Ni de acuerdo ni en desacuerdo

De acuerdo

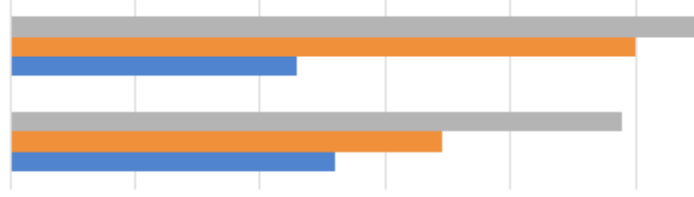

$\begin{array}{lllllll}0 & 20 & 40 & 60 & 80 & 100 & 120\end{array}$

- Solo pequeños grupos según la religión de pertenencia

En ocasiones Gran Grupo y en otras, pequeños grupos según religión de pertenencia

- Solo Gran Grupo independientemente de las religiones de pertenencia

Figura 1. Posición del alumnado respecto al tipo de agrupamiento en función de las religiones de pertenencia. 
Seguidamente, para conocer el tipo de agrupamiento con el que el alumnado se muestra más interesado, se han tenido en cuenta las siguientes tres variables, extraídas del grupo de ítems analizado: interés por la enseñanza a todos los alumnos juntos independientemente de su religión (solo gran grupo), interés por la enseñanza en parte a todos juntos y en parte separados por grupos (gran grupo y grupos pequeños) e interés por la enseñanza por separado en grupos según la religión de pertenencia (solo grupos pequeños).

Para el análisis de las diferencias se ha realizado un ANOVA de medidas repetidas con las cuatro variables intrasujetos. Se han encontrado diferencias significativas que afectan al grupo muestral para todas las variables de interés formativo actual ( $F$ $\left.[2,766]=31.427, p<.0001, \varepsilon^{2}=.076\right)$.

De los datos anteriores se desprende en primer lugar, como puede verse en la tabla (5), que hay acuerdo entre los alumnos respecto a que deberían estudiar Religión separados en grupos según la religión a la que pertenezcan (con un porcentaje acumulado del $54 \%, \mathrm{n}=208$ ), aunque también hay consenso en que la Religión debería ser enseñada en parte juntos y en parte en grupos en función de su pertenencia (en este sentido, el 33.3\% se posiciona neutral, $\mathrm{n}=128$ ).

En segundo lugar, encontramos que no hay manifestación de acuerdo ni de desacuerdo en cuanto a que la Religión debería ser enseñada a todos los alumnos juntos, independientemente de sus diferencias confesionales $(M=3.29)$. En este sentido, el alumnado indica que todos los temas necesarios están cubiertos por otras asignaturas en la escuela (por ejemplo, literatura, historia, etc.), no siendo necesario enseñar educación religiosa específica $(\mathrm{M}=3.2)$.

En tercer lugar, el alumnado no se muestra de acuerdo con la afirmación de que en la escuela no debería haber sitio para la religión (el 72\% del alumnado muestra algún grado de desacuerdo, $\mathrm{n}=277$ ).

Tabla 5. Respuesta registrada del alumnado de acuerdo con los diferentes modelos de enseñanza de religión en la escuela

\begin{tabular}{|c|c|c|c|}
\hline & $M$ & D.T. & $\mathbf{N}$ \\
\hline La Religión debería ser materia opcional. & 1,65 & 1,02 & 385 \\
\hline $\begin{array}{l}\text { Los alumnos/as deberían estudiar la Religión separados en grupos según la } \\
\text { religión a la que pertenecen. }\end{array}$ & 2,58 & 1,32 & 385 \\
\hline En la escuela no debería haber sitio para la religión. & 4,06 & 1,06 & 385 \\
\hline $\begin{array}{l}\text { La Religión debería ser enseñada a todos los alumnos/as juntos, } \\
\text { independientemente de las diferencias religiosas o confesionales de los alumnos. }\end{array}$ & 3,29 & 1,32 & 385 \\
\hline $\begin{array}{l}\text { Todos los temas necesarios están cubiertos por otras asignaturas en la escuela (ej. } \\
\text { literatura, historia, etc.) No es necesario enseñar educación religiosa específica. }\end{array}$ & 3,20 & 1,14 & 385 \\
\hline $\begin{array}{l}\text { La Religión debería ser enseñada en parte juntos y en parte en grupos, según la } \\
\text { religión a la que pertenecen los alumnos/as. }\end{array}$ & 2,68 & 1,15 & 384 \\
\hline
\end{tabular}


Finalmente, como refleja la siguiente tabla (6), los alumnos creen que deberían ser capaces de hablar y conversar sobre las cuestiones religiosas (el $64.7 \%$ muestra algún grado de acuerdo, $\mathrm{n}=249$ ), obtener un conocimiento objetivo de las diferentes religiones $(41.6 \%$ de acuerdo, $\mathrm{n}=160)$ y aprender sobre la importancia de la religión para resolver problemas en la sociedad $(37.4 \%$ de acuerdo, $n=144)$. No obstante, muestran neutralidad respecto a que deberían ser orientados hacia la creencia religiosa $(\mathrm{M}=3,1)$.

Tabla 6. Respuesta registrada del alumnado de acuerdo con sus deberes respecto a la religión

\begin{tabular}{lrrr}
\hline & M & D.T. & N \\
\hline Obtener un conocimiento objetivo de las diferentes religiones. & 2,26 &, 97 & 385 \\
\hline Ser capaz de hablar y conversar sobre las cuestiones religiosas. & 2,22 &, 92 & 385 \\
\hline $\begin{array}{l}\text { Aprender sobre la importancia de la religión para resolver problemas en la } \\
\text { sociedad. }\end{array}$ & 2,42 & 1,04 & 385 \\
\hline Ser orientados hacia la creencia religiosa. & 3,10 & 1,16 & 385 \\
\hline N válido (según lista) & & & 385 \\
\hline
\end{tabular}

\section{DISGUSIÓN Y CONGLUSIONES}

Tras el análisis de los datos se puede concluir que, con respecto a las experiencias de los estudiantes de Educación Secundaria Obligatoria con la religión dentro de las aulas, estos consideran que con ella aprenden a respetar a los demás, independientemente de sus creencias particulares, y no creen que el hecho de aprender sobre religiones genere conflictos en la institución escolar. Entienden, pues, que la religión y los actos voluntarios que de la misma derivan puedan formar parte de la vida académica, siendo necesario que se proporcionen espacios para ello, si bien consideran que la enseñanza doctrinal no debe ser motivo de faltas de asistencia a ciertas clases.

En cuanto a la opinión de los estudiantes respecto a los distintos posicionamientos de la escuela ante la religión, los datos señalan que no hay consenso sobre el uso de símbolos religiosos discretos, lo que tomamos como una sugerencia o invitación a hacer nuevas indagaciones al respecto.

La influencia que, a juicio de los sujetos de nuestro estudio, tiene la religión en sus procesos de aprendizaje es notoria. Esta, según ellos, puede favorecer la convivencia pacífica, generar nuevos aprendizajes sobre su religión, desarrollar sus propios puntos de vista, fraguar valores morales y hacer comprender mejor el presente y el pasado.

Por último, los datos obtenidos indican que los estudiantes de Secundaria Obligatoria están de acuerdo en que la Religión sea una materia opcional que se imparta en modelos didácticos flexibles que combinen actuaciones de enseñanza 
en gran grupo con otras en pequeños grupos, según la religión de pertenencia, y rechazan la idea de que deba mantenerse al margen de la institución escolar, pues esta les aporta saberes y destrezas como la capacidad de conversar sobre cuestiones religiosas, la de alcanzar un conocimiento objetivo de las distintas religiones o la de resolver problemas sociales.

Las conclusiones anteriores parecen reforzarse unas a otras. Todas ellas apuntan en la misma dirección: el pluralismo religioso es ampliamente aceptado por los jóvenes, y más que una fuente de conflicto es para ellos un motor de diálogo y convivencia. Las personas de religiones diferentes pueden estar juntas y en armonía. La coexistencia pacífica no solo ofrece la posibilidad de mejorar las relaciones interpersonales sino también los procesos de aprendizaje. Los actores políticos y sociales han de tomar conciencia de ello para el necesario crecimiento de la sociedad.

\section{REFERENCIAS}

Álvarez Castillo, José Luis; González González, Hugo y Fernández Caminero, Gemma (2012). El conflicto cultural y religioso. Aproximación etiológica. En Álvarez Castillo, José Luis y Essomba Gelabert, Miguel Angel (eds.). Dioses en las aulas, Barcelona: Graó, pp. 23-59.

Banks, James (2009). Diversity and Citizenship Education in Multicultural Nations. Multicultural Education Review, vol. 1, n.1, pp. 1-28. DOI: 10.1080/23770031.2009.11102861

Bertram-Troost, Gerdien; Schihalejev, Olga \& Neill, Sean (2014). Religious diversity in society and school: pupils' perspectives on religion, religious tolerance and religious education: an introduction to the REDCo research network. Religious Education Journal of Australia, vol. 30, n. 1, (en línea). Disponible en: https:// search.informit.com.au/documentSummary; $\mathrm{dn}=249207174213032$;res=IELHSS

Briones, Rafael (2010). ¿Y tú (de) quién eres? Minorías religiosas en Andalucía. Barcelona: Icaria.

Castilla, Carmen (2011). La diversidad religiosa en España: propuestas para el diálogo interreligioso. En: Castilla, Carmen (Coord.). El dialogo interreligioso. Iniciativas para la gestión de la diversidad religiosa. Granada: Comares, pp. 7-20.

Constitución Española, 1978. BOE núm. 311 de 29 de diciembre de 1978

Dietz, Gunther (2008). La educación religiosa en España: ¿Contribución al diálogo intercultural o factor de conflicto entre religiones? Revista Estudios sobre las Culturas Contemporáneas, vol. XIV, n. 28, pp. 11-46.

Dietz, Gunther; Rosón Lorente, Javier. y Ruiz Garzón, Francisca (2011). Homogeneidad confesional en tiempos de pluralismo religioso. Revista CPU-e, Revista de Investigación Educativa, n. 13, (en línea). Disponible en: http://www.uv.mx/cpue/ num13/inves/Dietz_homogeneidad confesional.html 
Estudio descriptivo de la educación religiosa ... - S. García-Segura y otros

European Commission (2011). Final Report Summary- REDCo (Religion in Education. A contribution to Dialogue or a factor of Conflict in transforming societies of European Countries) (en línea). Disponible en: https://cordis.europa.eu/docs/ publications/1238/123869721-6_en.pdf

Fernández Echeverría, José y García Castaño, Francisco Javier (2015). El desarrollo normativo que regula las aulas para escolares de nacionalidad extranjera. Profesorado: Revista de curriculum y formación del profesorado, 19(1), pp. 527-554.

Instrumento de ratificación del acuerdo entre el Estado Español y la Santa Sede sobre enseñanza y asuntos culturales. BOE núm. 300 de 15 de diciembre de 1979

Jackson, Robert (ed.) (2012). Religion, Education, Dialogue and Conflict. Perspectives on Religious Education Research. Routledge: New York.

Kay, William \& Ziebertz, Hans (2006). A nine-country survey of youth in Europe: selected findings and issues. British Journal of Religious Education, vol. 28, n. 2, 119-129. DOI: 10.1080/01416200500530748

López González, María (2012). Políticas europeas en relación con la formación en materia religiosa: la promoción del diálogo interreligioso y la educación intercultural. En Álvarez Castillo, José Luis y Essomba Gelabert, Miguel Angel (eds.). Dioses en las aulas. Barcelona: Graó, pp. 61-84.

Mijares Molina, Laura (2006). Aprendiendo a ser marroquíes. Inmigración y escuela en España. Madrid: Ediciones del Oriente y el Mediterráneo.

Ministerio de Educación, Cultura y Deporte (2013). Datos y cifras curso escolar 2013/2014 (I). Disponible en: http://www.mecd.gob.es/servicios-al-ciudadano$\mathrm{mecd} / \mathrm{en} / \mathrm{dms} / \mathrm{mecd} /$ servicios-al-ciudadano-mecd/estadisticas/educacion/ indicadores-publicaciones-sintesis/datos-cifras/Datos-y-Cifras-2013-2014-LR/ Datos\%20y\%20Cifras\%202013-2014 final.pdf

Ministerio de Educación, Cultura y Deporte (2017). Datos y cifras curso escolar 2017/2018. Disponible en: https://www.mecd.gob.es/servicios-al-ciudadano$\mathrm{mecd} / \mathrm{dms} / \mathrm{mecd} /$ servicios-al-ciudadano-mecd/estadisticas/educacion/indicadorespublicaciones-sintesis/datos-cifras/Datosycifras1718esp.pdf

Naidoo, Marilyn (2015). A REDCo Study: Learners' Perspectives on Religious Education and Religious Diversity in Catholic Schools in South Africa. Journal for the Study of Religion, vol. 28, n. 1, (en linea). Disponible en: https://www.jstor. org/stable/24805685

Ortega Ruiz, Pedro (2004). Cultura, valores y educación: principios de integración. En La educación en contextos multiculturales: diversidad e identidad. XIII Congreso Nacional y II Iberoamericano de Pedagogía. Madrid: Sociedad Española de Pedagogía, vol. 1, pp. 47-80.

Pedone, Claudia (2013). Las representaciones sociales en torno a la inmigración ecuatoriana a España. Íconos-Revista de Ciencias Sociales, n.14, pp. 56-66. 
UNESCO (2014). Constitución de la Organización de las Naciones Unidas para la Educación, la Ciencia y la Cultura (1945). Textos Fundamentales. Disponible en: http://unesdoc.unesco.org/images/0022/002269/226924s.pdf.

Valk, Pille; Bertram Troost, Gerdien; Friederici, Markus \& Béraud, Céline (eds.) (2009). Teenagers 'perspectives on the role of religion in their lives, schools and societies: A European quantitative study. Münster: Waxmann.

Weisse, Wolfram (coord.) (2009). Religión en educación: Contribución al diálogo. Sugerencias del proyecto de investigación REDCO para la política. Comisión Europea. Disponible en: https://www.awr.uni-hamburg.de/website-content/pdfsforschung/interreligioeser-dialog-zwid.pdf 
